\title{
Helicobacter pylori infection induces hyperammonaemia in Mongolian gerbils with liver cirrhosis
}

\author{
H Suto, T Azuma, S Ito, M Ohtani, M Dojo, Y Ito, Y Kohli, M Kuriyama
}

Second Department of Internal Medicine, Faculty of Medicine, Fukui Medical

University, Fukui,

Japan

H Suto

T Azuma

S Ito

M Ohtani

M Dojo

Y Ito

M Kuriyama

\section{Department of} Internal Medicine, Aiseikai Yamashina Hospital, Kyoto, Japan Y Kohli

Correspondence to: Dr T Azuma, Second Department of Interna Medicine, Faculty of Medicine, Fukui Medical University, 23-3

Shimoaizuki, Matsuoka-cho, Yoshida-gun, Fukui 910-1193, Japan. azuma@fmsrsa.fukui-med.ac.jp

Accepted for publication 14 November 2000

\begin{abstract}
Background and aims-We previously reported the effect of Helicobacter pylori eradication on hyperammonaemia in patients with liver cirrhosis. However, the role of $\mathrm{H}$ pylori as a cause of hyperammonaemia is controversial. We developed an animal model with liver cirrhosis and investigated the effect of $H$ pylori infection on hyperammonaemia.

Materials and methods-Five week old male Mongolian gerbils were inoculated orally with broth culture of $\boldsymbol{H}$ pylori. Forty eight gerbils were divided into four groups. Gerbils not inoculated with $H$ pylori were fed a commercial rodent diet (group A) or a choline deficient diet (group C). Gerbils inoculated with $H$ pylori were fed the commercial rodent diet (group B) or the choline deficient diet (group D). Blood ammonia levels of the femoral vein and portal vein were measured 30 weeks later.

Results-All gerbils fed the choline deficient diet developed liver cirrhosis with fatty metamorphosis. The survival rate of group D was significantly lower than that of the other groups. Systemic and portal blood ammonia levels in group $D$ were significantly higher than those in the other groups.
\end{abstract}

Conclusions-H pylori infection induces hyperammonaemia in gerbils with liver cirrhosis.

(Gut 2001;48:605-608)

Keywords: Helicobacter pylori; hyperammonaemia; liver cirrhosis; Mongolian gerbil; hepatic encephalopathy; choline deficient diet

Hyperammonaemia in patients with liver cirrhosis must be treated rapidly as it is one of the fatal complications of liver failure. It is thought to be caused by bacterial urease in the large intestine. ${ }^{1}$ Since the isolation of Helicobacter pylori from the human stomach, ${ }^{2}$ it has generally become accepted that $H$ pylori infection is implicated in the pathogenesis of gastroduodenal diseases. ${ }^{34} H$ pylori is also known to produce copious amounts of ammonia because of its strong urease activity, which is many times greater than that of urease positive enterobacteria. ${ }^{5-7}$ Previously, we reported the effect of $H$ pylori eradication on hyperammonaemia in two patients with liver cirrhosis. ${ }^{8}$ Subsequently, we found that eradication of $H$ pylori reduced systemic ammonia levels in patients with hyperammonaemia with diffuse $H$ pylori infection in the stomach. ${ }^{9}$ Some investigators also reported that $H$ pylori infection was likely to be associated with hyperammonaemia with cirrhosis. $^{10}{ }^{11}$ However, other workers have questioned whether or not the effect of $H$ pylori eradication therapy on hyperammonaemia is due to eradication of $H$ pylori or to the non-specific effect of antibiotic therapy on ammonia producing gut flora. ${ }^{12}$ The role of $H$ pylori as a cause of hyperammonaemia in patients with cirrhosis is thus controversial. There have been no reports on the effect of long term $H$ pylori infection in animals with liver cirrhosis.

Recently, Hirayama et al reported a technique for inoculation of $H$ pylori into the stomach of Mongolian gerbils. ${ }^{14}{ }^{15}$ In the gerbil model, $H$ pylori can form long term stable colonies in the stomach, which mimics the situation in humans in diseases such as chronic active gastritis, intestinal metaplasia, gastric ulcer, and gastric cancer. ${ }^{14-16}$ Therefore, using this model, we induced liver cirrhosis by feeding a choline deficient diet to gerbils and investigated the effect of long term $H$ pylor $i$ infection on hyperammonaemia associated with liver cirrhosis.

\section{Methods}

Forty eight specific pathogen free, five week old male Mongolian gerbils (MGS/Sea; Seac Yoshitomi, Ltd, Fukuoka, Japan) were housed in an air conditioned biohazard room for the purposes of $H$ pylori infection, with a 12 hour light/12 hour dark cycle. Animals were fed either a commercial rodent diet (CE-2; Clea Japan, Tokyo, Japan) or a choline deficient diet (CA-6; Clea Japan) and allowed free access to water.

H pylori (ATCC43504; American Type Culture Collection) was grown in brucella broth containing $10 \% \mathrm{v} / \mathrm{v}$ horse serum for 48 hours at $37^{\circ} \mathrm{C}$ under microaerobic conditions (15\% $\mathrm{CO}_{2}$ ) and high humidity with shaking (120 $\mathrm{rpm})$. The broth culture $(500 \mu \mathrm{l})$ of $\mathrm{H}$ pylori $\left(4 \times 10^{8} \mathrm{CFU} / \mathrm{ml}\right)$ was inoculated orally, using a feeding needle, after the gerbils had been fasted for 24 hours.

Forty eight gerbils were divided into four groups (12 in each group). Gerbils not inoculated with $H$ pylori were fed a commercial rodent diet (group A) or a choline deficient diet (group C). Gerbils inoculated with $H$ pylori were fed the commercial rodent diet (group B) or the choline deficient diet (group D). All gerbils were observed for 30 weeks to investigate 
survival. After this, six gerbils each from groups $\mathrm{A}, \mathrm{B}$, and $\mathrm{C}$ and the five surviving gerbils from group $\mathrm{D}$ were sacrificed under anaesthesia with pentobarbiturate for examination of ammonia levels.

Blood $(50 \mu \mathrm{l})$ was obtained from the right femoral vein and portal vein, and blood ammonia levels were quantitated using an Amicheckmeter (AA-4120; Kyoto Daiichi Kagaku, Kyoto, Japan) which requires only $20 \mu \mathrm{l}$ of blood. Blood ammonia levels were expressed in $\mu \mathrm{mol} / 1$. If necessary, blood samples were diluted with saline, as previously described. ${ }^{17}{ }^{18}$ In addition, blood was obtained from the inferior caval vein for blood chemistry, and serum was separated by centrifugation and measured by an automated chemistry analyser (AU-5200; Olympus Japan, Tokyo, Japan). Half of the stomach and liver of the gerbils were fixed in $10 \%$ phosphate buffered formalin, embedded in paraffin, and sectioned into $4 \mu \mathrm{m}$ samples. The stomach was stained with haematoxylin-eosin and Giemsa, and the liver with haematoxylin-eosin and Azan for histopathological examination. The remaining half of the stomach was homogenised with $10 \mathrm{ml}$ of phosphate buffered saline. An aliquot $(100 \mu \mathrm{l})$ of dilution was cultured in TSA-II agar plate (Nippon Becton Dickinson, Tokyo, Japan) for four days at $37^{\circ} \mathrm{C}$ under microaerobic conditions $\left(\mathrm{O}_{2} 5 \%, \mathrm{CO}_{2} 15 \%, \mathrm{~N}_{2}\right.$ $80 \%)$.

Fisher's protected least significance difference was used to test the significance of differences between ammonia levels in the different groups. The log rank test was used to compare survival rates of the different groups. The Student's $t$ test was used to compare the results of blood chemistry and body weight. A p value $<0.05$ was considered significant.

\section{Results}

All Mongolian gerbils fed the choline deficient diet (groups C and D) developed liver cirrhosis with fatty metamorphosis (fig 1A), while all gerbils fed a commercial rodent diet (groups $\mathrm{A}$ and B) had a normal liver (fig 1B). All gerbils in groups $\mathrm{C}$ and $\mathrm{D}$ had slight ascites and the liver parenchyma was subdivided into small pseudolobules. Hepatic cells contained a large amount of fat and were markedly swollen. The sinusoidal lumen was conspicuously compressed. The histological finding of the liver was considered to be stage IIIB in all gerbils in groups C and D, according to Hoffbauer's report. ${ }^{19}$ There were no significant differences between the histological findings of the liver in groups C and D. There were no cases of hepatocellular carcinoma or other cancers in any group.

The gerbils in groups $\mathrm{A}$ and $\mathrm{C}$ did not have gastric ulcers or gastritis. In all gerbils in groups $\mathrm{B}$ and $\mathrm{D}$, which had been inoculated with $H$ pylori, the oedematous thickening of the mucosa was generated in the antrum and adjacent part of the corpus, and the lamina propria of the antrum showed gastritis with massive mononuclear cells and neutrophil infiltration with superficial erosions. In addition, two animals each in groups $\mathrm{B}$ and $\mathrm{D}$ had gastric ulcers. $H$ pylor $i$ was detectable either by culture
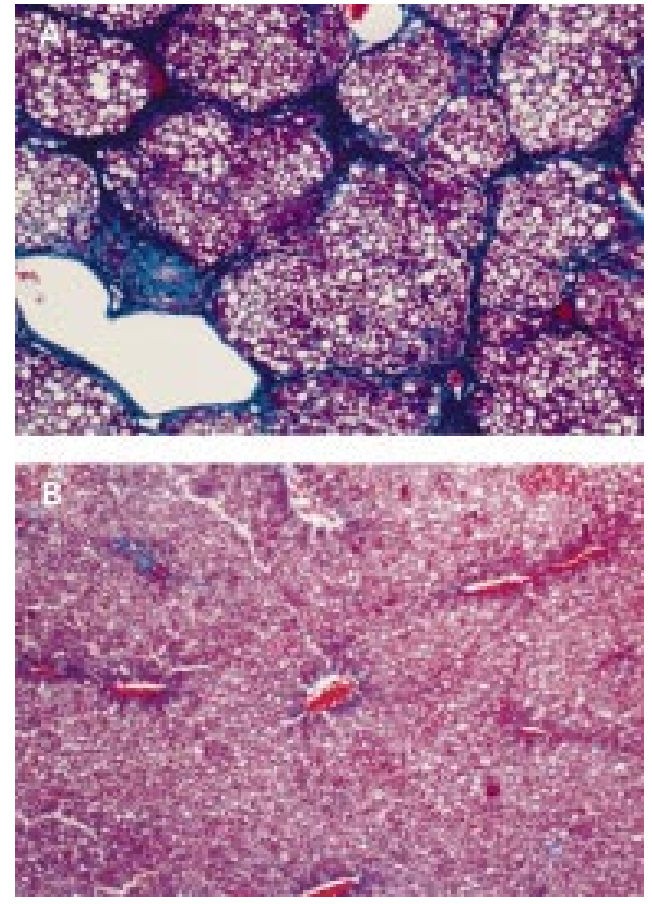

Figure 1 Histological findings showing liver cirrhosis with fatty metamorphosis in Mongolian gerbils fed a choline deficient diet (A: Azan stain, $\times 100)$, and normal liver in gerbils fed a commercial rodent diet (B: Azan stain, $\times 100)$.

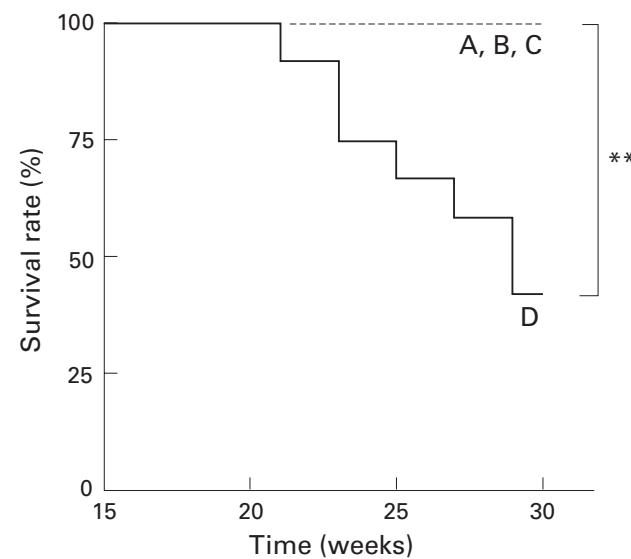

Figure 2 Survival rate of each group of Mongolian gerbils. The survival rate of group $D$ was significantly lower than that of the other groups $\left({ }^{*} p<0.01\right)$.

or by histological findings in the gastric mucosa of all gerbils in groups B and D; however, it was not detected in gerbils in groups $\mathrm{A}$ and $\mathrm{C}$. The number of $H$ pylori colonies in each infected gerbil was approximately $6.0 \quad \log \mathrm{CFU} /$ stomach.

All Mongolian gerbils in groups A, B, and C survived for 30 weeks. However, in group D, only five of 12 gerbils survived for 30 weeks. The survival rate was $41.7 \%$ in group D (fig 2 ), which was significantly lower than that in the other groups $(\mathrm{p}<0.01)$.

Ammonia levels in blood from the right femoral vein of groups A, B, C, and D were 105 (29), 128 (17), 136 (16), and 180 (30) $\mu \mathrm{mol} / 1$, respectively (fig 3 ). Ammonia levels in blood from the right femoral vein of group D were significantly higher than those of the other groups $(p<0.01)$. Blood ammonia levels in group $\mathrm{C}$ were significantly higher than those of 


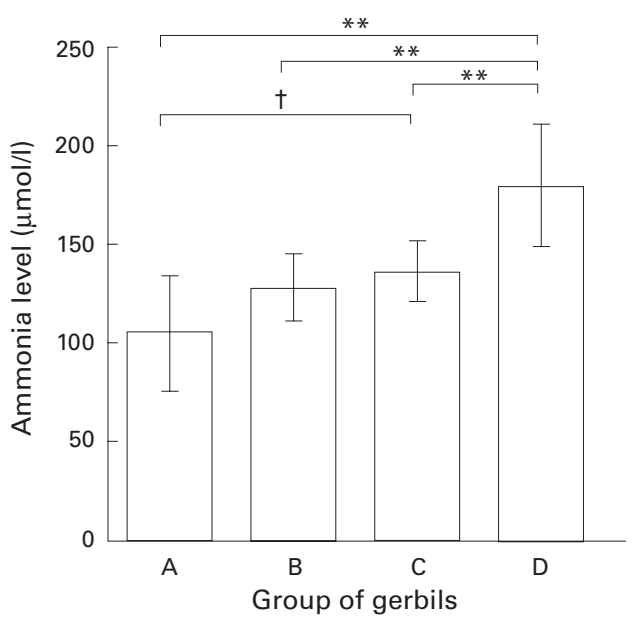

Figure 3 Systemic ammonia levels in the groups of Mongolian gerbils. Ammonia levels in blood from the femoral vein of group $D$ were significantly higher than those of the other groups $\left({ }^{\star} p<0.01\right)$. Blood ammonia levels in group $C$ were significantly higher than those of group $A$ $(t p<0.05)$.

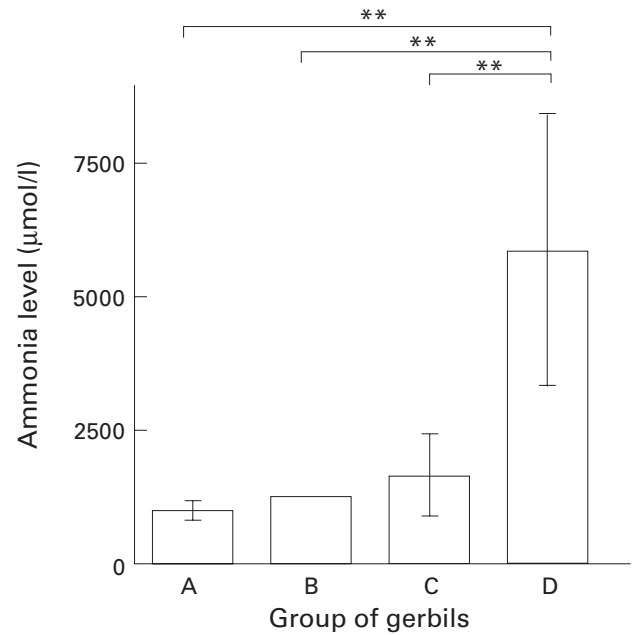

Figure 4 Portal ammonia levels in the groups of Mongolian gerbils. Ammonia levels in blood from the portal vein of group $D$ were significantly higher than those of the other groups $\left({ }^{\star \star} p<0.01\right)$

group A $(p<0.05)$. Ammonia levels in blood from the portal vein of groups A, B, C, and D were 966 (199), 1240 (62), 1659 (780), and 5936 (2570) $\mu \mathrm{mol} / 1$, respectively (fig 4). Ammonia levels in blood from the portal vein of group $\mathrm{D}$ were significantly higher than those of the other groups $(\mathrm{p}<0.01)$.

Serum alanine aminotransferase, albumin, total bilirubin, and cholinesterase were examined in gerbils in groups $\mathrm{C}$ and $\mathrm{D}$, which were fed a choline deficient diet. In groups C and D, mean serum alanine aminotransferase was 80 (54) and 119 (23) IU/l, mean serum albumin was $3.2(0.2)$ and $3.1(0.5) \mathrm{g} / \mathrm{dl}$, mean serum total bilirubin was 0.1 and $0.1 \mathrm{mg} / \mathrm{dl}$, and mean serum cholinesterase was 5077 (896) and 5413 (483) IU/1, respectively. Mean body weight was 53.9 (2.7) g and 53.2 (5.0) $\mathrm{g}$ in groups $\mathrm{C}$ and $\mathrm{D}$, respectively. There was no significant difference between groups C and D in blood chemistry or body weight.

\section{Discussion}

In the present study, systemic blood ammonia levels in group $\mathrm{C}$ were significantly higher than those in group A and systemic blood ammonia levels in group $\mathrm{D}$ were significantly higher than those in the other groups. Portal ammonia levels in group D were significantly higher than those of the other groups. All Mongolian gerbils fed the choline deficient diet (groups C and D) developed liver cirrhosis and there were no significant differences between groups C and $\mathrm{D}$ in liver function or pathological results. These findings suggest that $H$ pylori infection produces copious amounts of ammonia in the stomach and causes marked elevation of portal ammonia levels in gerbils with liver cirrhosis. In addition, $H$ pylori influences systemic ammonia levels in gerbils with liver cirrhosis.

A choline deficient diet induced liver cirrhosis with fatty metamorphosis in rats in previous studies. ${ }^{19-22}$ Hartroft initially reported that rats developed pathological fatty cirrhosis if fed a choline deficient diet. ${ }^{20}{ }^{21}$ Hoffbauer reported that after the choline deficient diet was begun, the evolution from fatty liver to cirrhotic liver could be recognised in the rat liver. ${ }^{19}$ Wada et al reported that the mechanism of portal hypertension in this model was similar to that of carbon tetrachloride induced rat cirrhosis and to that of portal hypertension in humans. ${ }^{22}$ It was confirmed that a choline deficient diet could also induce liver cirrhosis in gerbils in the present study.

The prognosis of group D was significantly worse than that of group C. During this study, seven of 12 gerbils in group $\mathrm{D}$ died. In the liver, histological findings that could be determined in these gerbils showed similar or earlier stage findings, compared with gerbils that survived. In addition, systemic ammonia levels in the dying gerbils were very high and in our previous study most showed more than 500 $\mu \mathrm{mol} / 1$ in orbital sinus blood before they died. Also, there were no significant differences between $H$ pylori infected and uninfected gerbils with cirrhosis in terms of liver histological findings and blood chemistry at 16 or 24 weeks in those studies. Therefore, we speculated that the difference in ammonia levels in these two groups became more marked as the duration of receiving the choline deficient diet became longer and the liver damage became more severe. The gerbils that died in group D were considered to have developed hepatic encephalopathy with hyperammonaemia. Hepatic encephalopathy leads to disturbances of consciousness and occasionally to hepatic coma. Furthermore, it may cause death in small animals as they are not treated with conventional therapy used in humans. $H$ pylori infection may also influence the prognosis in gerbils with liver cirrhosis.

Hepatic encephalopathy is precipitated by compounds such as urea which can contribute to elevated ammonia levels frequently present in symptomatic patients. ${ }^{23}$ Blood ammonia levels and symptomatology increase as liver function declines. ${ }^{24}$ Most ammonia is from the gut where venous ammonia levels greatly exceed systemic levels. ${ }^{25}$ Summerskill and Wolpert 
showed that gastric mucosal urease activity was much higher than colonic mucosal urease activity, and most of the gut derived ammonia was generated by luminal or faecal urease, ${ }^{1}$ although the quantity of ammonia that moved from the stomach to the colon was not considered in their study. Rappoport and Kern showed that gastric mucosal urease activity of patients with cirrhosis was significantly greater than that of normal subjects. ${ }^{26}$ In addition, increased gastric ammonia has been reported to be an important source of systemic ammonia. ${ }^{27}$

Previous studies on the effect of $H$ pylori in patients with hyperammonaemia showed such a role ${ }^{8-11}$ while other studies showed no such role. ${ }^{12}{ }^{13}$ In humans, most patients had already been treated to various degrees with conventional therapy - for example, a low protein diet, antibiotics, lactulose, and branched chain enriched amino acid solution. These conventional therapies may prevent elucidation of the role of $H$ pylori infection in hyperammonaemia, although such patients need to be treated adequately. Therefore, animal models may be preferable for studying the effect of $H$ pylori infection on hyperammonaemia as animals have not been treated.

Previously, we examined the effect of instillation of $H$ pylori into the stomach on systemic ammonia levels in rats with cirrhosis induced by carbon tetrachloride. ${ }^{17}$ The study revealed that ammonia levels in portal and venous blood significantly increased after instillation of $10^{7}$ bacteria CFU in the stomach of cirrhotic rats, and suggested that the ammonia produced by $H$ pylori induced hyperammonaemia when this organism was widely distributed and was present in large numbers in the stomach, particularly in the presence of cirrhosis. The study described the short term effect of instillation of $H$ pylori into the rat stomach because $H$ pylori cannot form colonies in the stomach. ${ }^{28}$ In the present study, the effect of long term $H$ pylori infection in an animal model of hyperammonaemia was demonstrated.

Kusuhara et al reported on eradication therapy for $H$ pylori infected gerbils ${ }^{29}$ and we also tried eradication therapy, consisting of omeprazole, clarithromycin, and amoxicillin, in another group of $H$ pylor $i$ infected gerbils with liver cirrhosis. However, most of these gerbils died soon after dosing because of liver failure due to drug induced liver injury, although the dose was smaller than previously reported. The establishment of an appropriate dose of these drugs was difficult for gerbils with liver cirrhosis, although trials of various dosages were performed. Therefore, we could not investigate the effect of $H$ pylori eradication in infected gerbils with liver cirrhosis. However, the present study clearly indicated that the urease of $H$ pylori as well as intestinal bacterial urease caused elevation of systemic and portal ammonia levels in gerbils with liver cirrhosis. We conclude that $H$ pylori infection induces hyperammonaemia in gerbils with liver cirrhosis.

1 Summerskill WHJ, Wolpert E. Ammonia metabolism in the gut. Am f Clin Nutr 1970;23:633-9.

2 Warren JR, Marshall BJ. Unidentified curved bacilli on gastric epithelium in active chronic gastritis. Lancet 1983;1: 1273-5.

3 Sipponen P, Hyvarinen H. Role of Helicobacter pylori in the pathogenesis of gastritis, peptic ulcer, and gastric cancer. Scand F Gastroenterol 1993;28(suppl 1):3-6.

4 Wotherspoon AC, Ortez-Hidalgo C, Diss TC. Helicobacter pylori associated gastritis and primary B-cell gastric pylori associated gastritis and prim
lymphoma. Lancet 1991;338:1175-6.

5 Mobley HLT, Cortesia MJ, Rosenthal LE, et al. Characterization of urease from Campylobacter pylori. $\mathcal{F}$ Clin Microbiol 1988;26:831-6.

6 Dunn BE, Campbell GP, Perez Perez GI, et al. Purification and characterization of urease from Helicobacter pylori. $\mathcal{F}$ Biol Chem 1990;265:9464-9.

7 Ito S, Kohli Y, Kato T, et al. Differences in urease activity in live Helicobacter pylori cultured from patients with gastroduodenal diseases. Eur $\mathcal{F}$ Gastroenterol Hepatol 1995; 7(suppl 1):S83-8.

8 Ito S, Miyaji H, Azuma T, et al. Hyperammonaemia and Helicobacter pylori. Lancet 1995;346:124-5.

9 Miyaji H, Ito S, Azuma T, et al. Effects of Helicobacter pylori eradication therapy on hyperammonaemia in patients with liver cirrhosis. Gut 1997;40:726-30.

10 Zullo A, Rinaldi V, Folino S, et al. Helicobacter pylori urease inhibition and ammonia levels in cirrhotic patients. Am $\mathcal{F}$ Gastroenterol 1998;93:851-2.

11 Dasani BM, Sigal SH, Lieber CS. Analysis of risk factors for chronic hepatic encephalopathy: the role of Helicobacter pylori infection. Am f Gastroenterol 1998;93:726-31.

12 Quero JC, Hartmann IJC, de Rooij F, et al. Hyperammonaemia and Helicobacter pylori. Lancet 1995;346:713-14.

13 Plevris JN, Morgenstern R, Hayes PC, et al. Hyperammonaemia in cirrhosis and Helicobacter pylori infection. Lancet 1995;346:1104.

14 Hirayama F, Takagi S, Yokoyama Y, et al. Establishment of gastric Helicobacter pylori infection in Mongolian gerbils. $\mathcal{F}$ Gastroenterol 1996;31 (suppl 9):24-8.

15 Hirayama F, Takagi S, Kusuhara H, et al. Induction of gastric ulcer and intestinal metaplasia in Mongolian gerbils infected.

16 Watanabe $\mathrm{T}$, Tada $\mathrm{M}$, Nagai $\mathrm{H}$, et al. Helicobacter pylori infection induces gastric cancer in Mongolian gerbils. Gastroenterology 1998;115:642-8.

17 Ito S, Kohli Y, Kato T, et al. Significance of ammonia produced by Helicobacter pylori. Eur $\mathcal{f}$ Gastroenterol Hepatol 1994;6:167-74

18 Yoneda K, Katayama Y, Katayama H, et al. Invention and evaluation of a new measuring system for blood ammonia by Drychemistry (in Japanese). F Clin Lab Inst Reag 1988; 11:105-9.

19 Hoffbauer FW. Fatty cirrhosis in the rat. I. A method of grading specimens. Arch Pathol 1959;68:160-70.

20 Hartroft WS. Accumulation of fat in liver cells and in lipodiastaemata preceding experimental dietary cirrhosis. Anat Rec 1950;106:61-87.

21 Hartroft WS, Ridout JH. Pathogenesis of the cirrhosis produced by choline deficiency. Am ₹ Pathol 1951;27:951-89.

22 Wada K, Fujimoto K, Fujikawa Y, et al. Sinusoidal stenosis as the cause of portal hypertension in choline deficient diet induced fatty cirrhosis of the rat liver. Acta Path fpn 1974; 24:207-17.

23 Phillips GB, Schwartz R, Gabuzda GJ Jr, et al. The syndrome of impending hepatic coma in patients with cirrhosis of the liver given certain nitrogenous substances. $N$ Engl f Med 1952;247:239-46.

24 Rikkers L, Jenko P, Rudman D, et al. Subclinical hepatic encephalopathy: detection, prevalence, and relationship to nitrogen metabolism. Gastroenterology 1978;75:462-9.

25 Cordoba J, Blei AT. Treatment of hepatic encephalopathy. Am F Gastroenterol 1997;92:1429-39.

26 Rappoport WJ, Kern F. Gastric urease activity in normal subjects and in subjects with cirrhosis. $\mathcal{f}$ Lab Clin Med subjects and in

27 Webster LT, Gabuzda GJ. Relation of azotemia to blood ammonium in patients with hepatic cirrhosis. Arch Intern Med 1959;103:15-22.

28 Ferrero RL, Lee A. The importance of urease in acid protection for the gastric colonizing bacteria Helicobacter pylori and Helicobacter felis sp.nov. Microb Ecol Health Dis 1991;4:121-34.

29 Kusuhara H, Hirayama F, Matsuyuki H, et al. Evaluation of combined antibiotic-omeprazole therapies in Helicobacter pylori-infected Mongolian gerbils. F Gastroenterol 1998;33: 14-17. 